\title{
Exploration on enterprise management strategy under the guidance of lean concept
}

\author{
Liao Kunke ${ }^{1, a}$
}

${ }^{1}$ Chongqing College of Electronic Engineering, Chongqing, 401331

Keywords: lean concept; enterprise; operating and management; management strategy

\begin{abstract}
Lean management ideas originally generated in lean production. Lean production concept has gradually form lean management ideas through continuous application and development. This has gradually become an enterprise management strategy in modern society, and has a certain positive impact on the realization of the strategic objectives of enterprise operating and management. This paper starts from the lean concept to make systematic analysis of enterprise management strategy model under the guidance of the lean concept, so as to offer a good support for the improvement of strategic level of enterprise operating and management. The lean management idea was first proposed by MIT professor for the analysis of Japanese Toyota JIF production model based on the survey and comparative research after putting forward the learning and upgrading strategy based on the investigation and comparison stud. The core idea of the lean management is to use the minimum resources to create the greatest value through the scientific management. The lean management concept has the corresponding positive impact on practical application of the enterprise integrated management, which is helpful to promote ultimate realization of objectives of enterprise management strategy. At current stage, the lean concept has begun to extend to other industries beyond manufacturing sector through continuous development, and has been widely respected in the practical application process. Therefore, China's enterprises can try to introduce lean concept in the process of seeking modern management in the new era, thus to offer the appropriate support for the implementation of enterprise management strategy.
\end{abstract}

\section{Introduction}

The lean concept has a certain positive impact on the improvement of the enterprise integrated management. In the new period, China's enterprise should analyze the application of lean concept in order to further improve the management level and explore the road of modernization construction, thus to offer scientific guidance for enterprise management. Specifically, the importance of incorporating lean concept into enterprise management is manifested in the following aspects.

Currently, due to the influence of economic globalization, the market scale in the process of China's economic development is increasing, enterprise competition is more intense. The enterprise not only needs to deal with the impact of foreign companies, but also needs to face the competition of domestic industry, which has caused a great pressure for enterprise survival and development. Especially for the manufacturing enterprises at the bottom of the industrial chain, the enterprise profit shows the downward trend year by year under the influence of increasing market competition. The increase in labor costs and deepened price transparency have produced a negative impact on the development of processing industry. In such social context, enterprise should explore the lean production concept and implement the intensive management in order to achieve sustained and stable development to win in the fierce market competition, thus to minimize waste. So that limited resources to produce greater economic benefits, and provide good support for healthy development 
of enterprise. Meanwhile, with the intensification of social competition, the real demand for enterprise to retain talent has been increasingly stronger, and the necessity of exploring lean management idea in enterprise has been gradually highlighted. Enterprises can only play the talent advantage and achieve the full development by attracting talent and retaining talents via lean management.

With the intensification of social talent competition and the rising of labor costs, in the condition that the manufacturing industry in surrounding countries has been developed constantly, the transformation and upgrading of China's manufacturing in many areas are facing enormous pressure, there's great difficulty for transformation and upgrading, which is with high degree of risk. Only enterprises form a profound field of the market and have good market responsiveness, and then the enterprises are able to achieve a good transition. The lean management concept not only can provide the corresponding guidance to production management, but also can be appropriately used in the internal management, ERP information construction and marketing of the enterprise, which can further realize the refinement of internal management of the enterprises, promoting the overall management level within the enterprise to be significantly improved. In this regard, under the support of the lean management concept, when enterprises obtain certain degree of market observation and internal operational capacity, they can find the appropriate opportunity in the market in a timely manner to seize the opportunity to achieve transformation and upgrading. In this way, the enterprises can effectively improve their position in the industry chain, and achieve the maximum economic and social benefits.

In the new period, the CPC Central Committee put forward the strategic goal of the great Chinese dream combined with the development of China's national construction. The realization of the great Chinese dream of the national rejuvenation is inseparable from support of enterprise funds and culture. Only the national rejuvenation can smoothly achieve the Chinese dream under the good financial support and cultural guidance. However, at current stage, most of state-owned enterprises in China have yet not established the scientific management concept in operating and management, there is phenomenon of extravagance and waste, duplication and capital occupation problem is relatively serious ${ }^{[1]}$. Therefore, in order to ensure the realization of the Chinese dream according to these problems to provide good support for construction of conservation-oriented society. The state-owned enterprises should introduce the concept of lean management concept to effectively enhance management of enterprise funds, production and internal operations, thus to increase enterprise revenue, and then create greater economic benefits. This can provide the good support for the construction of other aspects in China. Meanwhile, analysis is conducted from another point of view, and lean management idea is also a special form of culture. Under the background of comprehensive deepening reform in the 18th National Congress, enterprise should offer the good support for the construction of the enterprises, thus to change backward mode of production and management in the past, exploring the people-oriented, unity and also the cooperation management. This can provide good support for enterprise construction, and promote the realization of great Chinese dream.

\section{Implementation of enterprise management strategy planning under guidance of lean concept}

Under the guidance of leaching concept, implementation of enterprise management strategy involves four links. Therefore, the specific implementation strategy should be developed according to different links. 
Under the guidance of the lean concept, after enterprise completes the development of strategic objectives, in order to ensure the implementation of strategic objectives, enterprise should first decompose strategic objectives, which is divided into quarterly strategic objectives, monthly strategic objectives, etc. The objectives of enterprise management strategy can also be divided into the strategic objectives of various departments, and then the relevant responsibilities of strategic objectives are decomposed into individuals of enterprises in accordance with different departments. In this way, the enterprise employees can form a clear understanding of the realization of objectives of enterprise management strategy, and guide the enterprise employees to have an organic combination between personal development goals, and contribute the corresponding strength for the realization of enterprise strategic objectives while realizing the personal development. In this way, the reasonable decomposition of enterprise strategic objectives under the guidance of the lean concept can provide corresponding support for implementation of enterprise management strategy. [2]

After rational decomposition of objectives of enterprise management, it is necessary to guarantee that the reasonable strategic implementation plan based on the guidance of lean concept, thus to guarantee the orderly promotion of implementation of the strategic objectives to make a strategic implementation plan based on the guidance of lean concept. In specific operation, enterprise continues to cycle and forward in four links of "plan, do, check, and modify" via development of dynamic PADC model. It can be said that in the integrated management of enterprise, the development of implementation plan of management strategy is essentially a formulation of relevant work and sufficient resources of the enterprise management strategy. Combined with the actual situation to carry out the relevant management strategy activities in plan, enterprise can move forward to the management strategy objectives step by step. Therefore, enterprise management strategy plan has played an important role in the process of enterprise development. In order to obtain the good development, it's essential to maintain a high priority on the strategic implementation plan, and actively develop a reasonable strategic implementation plan to provide a good support for the improvement of strategic management level. After the comprehensive analysis of enterprise development, it's found that the following problems should be considered in the process of develop the management strategy plan.

First is the rational use of Gantt chart in the process of developing the strategic planning, the corresponding work cycle should be designed by combined with the different work model, and limit the starting time ${ }^{[3]}$. Since the implementation of some management plans requires to be based on the completion of other management work, and realization of management strategy objectives is subject to practice restrictions, so the use of Gantt chart for restriction can allow enterprise employees to clear about their own responsibility, and urge enterprise employees to consciously and efficiently complete post jobs. Second, a reasonable implementation plan should be developed according to different strategic objectives, thus to ensure to provide appropriate guidance for the implementation of each management strategy, thus to regulate the behavior of employees and offer the corresponding guarantee for the orderly promoted strategic planning. Third is to clear about the responsible people with different action steps, and strengthen sense of responsibility of management staff and grassroots employees, thus to provide the good support for effective implementation of the strategic planning. Only in this way, the role of the enterprise management strategy plan can be fully played to contribute the appropriate strength for promoting the realization of objectives of enterprise management strategy. 
Enterprise management strategy planning can make the correct guidance for all work of enterprise. In order to ensure the smooth realization of strategic objectives, there should be the reasonable limitation of the implementation content under the guidance of the lean concept in order to guarantee the smooth realization of the strategic objectives, thus to promote t enterprise strategic objectives to be completed efficiently. Specific analysis of enterprise strategic content should be defined and implemented from the perspective of lean management. First, attention should be paid to reduce the cost and gradually reduce the inventory in the enterprise management work, thus to avoid the emergence of waste problems in maximum. Second, the enterprise should develop appropriate measures to reduce the production step by step, to promote their own inventory asset turnover rate to be further enhanced, thereby driving the increase in cash flow and effective development of capital efficiency, offering corresponding support for the realization of strategic objectives. Furthermore, enterprises should effectively use their potential capacity and potential resources to effectively expand the opportunity rate in operating and management work, and introduce the richer business resources for providing the resource protection to the enterprise integrated management ${ }^{[4]}$. Finally, enterprises should focus on reforming their operating and management ideas, and incorporate the human-based management idea, thus to create more value via the scientific management to create good condition for enterprise development. In this way, the lean management system is constructed under the guidance of lean concept can guarantee content of enterprise management strategy to be well defined, and the enterprise staffs are organized to be involved in the practice of the enterprise management strategy, thus to offer the comprehensive support for the realization of enterprise management strategy.

In the implementation process of enterprise management strategy plan, there should also be continuous evaluation of the plan combined with the actual implementing results. If there's a greater change of some important influence factors appear during the implementation, there should be reasonable adjustment and improvement of the strategic implementation plan, so as to ensure that the implementation plan of the management strategy can offer scientific guidance for realization of the strategic objectives, thus to make enterprise strategic behavior to be always on the correct track, and provide good support for the efficient realization of the strategic goal ${ }^{[5]}$. It can be seen that the evaluation and improvement of the strategic implementation plan is also more important content that requires the attention of the enterprise managers.

\section{Conclusion}

In short, the realization of enterprise management strategy objectives is a systematic project, which requires the management personnel to continue to introduce advanced management ideas, making innovation of management work, requiring departments of the enterprises to actively cooperate with the integrated management. Therefore, it's with certain value for the enterprise to introduce lean management system in the process of gradual promotion of the management strategy. This can provide the good support for the realization of the strategic goal via the lean management, and ensure that the enterprise occupies the corresponding cost advantage in the overall market competitive environment, talent advantage and management advantages, and others, thus to provide a solid guarantee for the enterprise modernization construction and development. 


\section{Reference}

[1] Li Zhuoxuan, Ding Ling, Strategic Research on Optimized Enterprise Production Mode under Lean Concept [J], Technology entrepreneurship monthly, 2016,29 (6): 57-58,63.

[2] Zhao Rongrong, Lean Management of KT Company Product Development at Pilot Stage [D], Tianjin Normal University, 2015

[3] Wang Xiaoxia, Xu Guang, On How to Use Lean Concept to Enhance Enterprise Competitiveness [J], Small and medium-sized enterprise management and technology, 2015 (10): $1-1$.

[4] Task Group of North China Electric Power Co., Ltd of National Grid, "Lean 365" management law of innovative power generation enterprise [J], China Power Enterprise Management, 2014 (1): 68-70.

[5] Li Guodong, Promote Enterprise Management Progress with Lean Management [J], Chinese and foreign enterprises, 2016 (5): 10. 